Canad. Math. Bull. Vol. 20 (1), 1977

\title{
A NOTE ON THE CATEGORY OF THE TELESCOPE
}

\author{
BY \\ K. A. HARDIE
}

Let $X$ be an infinite connected $C W$-complex which is the union of an increasing sequence of subcomplexes $X_{r}$. Let cat $X$ denote the LusternikSchnirelmann category of $X$, normalized to take the value 0 on contractible spaces. Suppose that cat $X_{r} \leq k(r \geq 1)$. In his problem list [1], T. Ganea proved that cat $X \leq 2 k+1$ and asked (Problem 5) whether this is the best possible upper bound. The purpose of this note is to prove that cat $X \leq 2 k$.

As pointed out by Ganea, we may replace $X$ by the telescope $Y=$ $\cup(r \geq 1) X_{r} \times[r-1, r]$. The required inequality will be obtained by representing $Y$ as a double mapping cylinder and applying the main result of [2].

Let $W=\bigvee(r \geq 1) X_{r}, A=\bigvee(r \geq 1) X_{2 r-1}, B=\bigvee(r \geq 1) X_{2 r}$ be wedges as indicated and let $f: W \rightarrow A$ map $X_{2 r}$ by inclusion into $X_{2 r+1}$ and map $X_{2 r-1}$ identically. Similarly let $g: W \rightarrow B$ map $X_{2 r-1}$ by inclusion into $X_{2 r}$ and map $X_{2 r}$ identically. Then certainly $Y$ is homeomorphic to the double mapping cylinder $Z=Z(f, g)$. By [2; (2)], we have cat $Z \leq$ cat $X+\max$ (cat $A$, cat $B$ ). But cat $W \leq k$, cat $A \leq k$ and cat $B \leq k$. Hence cat $Y=$ cat $Z \leq 2 k$.

Acknowledgement. Grants to the Topology Research Group from the University of Cape Town and the South African Council for Scientific and Industrial Research are acknowledged.

\section{REFERENCES}

1. T. Ganea, Some problems on numerical homotopy invariants. Symposium on Algebraic Topology, Battelle Seattle Research Centre 1971, Lecture Notes in Mathematics 249, Springer-Verlag, Berlin, 1971.

2. K. A. Hardie, On the category of the double mapping cylinder, Tôhoku Mathematical Journal, 25 (1973), 355-358.

Department OF MATHEMATICS

UNIVERSITY OF CAPETOWN

RONDEBOSCH 7700

RePUblic of South AfricA

Received by the editors June 10, 1976. 